\title{
LIMIT VARIETIES OF APERIODIC MONOIDS WITH COMMUTING IDEMPOTENTS
}

\author{
S.V.GUSEV
}

\begin{abstract}
A variety of algebras is called limit if it is non-finitely based but all its proper subvarieties are finitely based. A monoid is aperiodic if all its subgroups are trivial. We classify all limit varieties of aperiodic monoids with commuting idempotents.
\end{abstract}

\section{INTRODUCTION AND SUMMARY}

A variety of algebras is called finitely based if it has a finite basis of its identities, otherwise, the variety is said to be non-finitely based. Much attention is paid to studying of finitely based and non-finitely based varieties of algebras of various types. In particular, the finitely based and non-finitely based varieties of semigroups and monoids have been the subject of an intensive research (see the surveys $[14,15]$ ).

A variety is hereditarily finitely based if all its subvarieties are finitely based. A variety is called a limit variety if it is non-finitely based but every its proper subvariety is finitely based. The limit varieties play an important role because every non-finitely based variety contains some limit subvariety by Zorn's lemma. It follows that a variety is hereditarily finitely based if and only if it does not contain any limit variety. So, if one manages to classify all limit varieties within some class of varieties then this classification implies a description of all hereditarily finitely based varieties in this class.

We consider varieties of monoids as semigroups equipped with an additional 0-ary operation that fixes the identity element. A monoid is aperiodic if all its subgroups are trivial. The article is devoted to study the limit varieties within the class $\mathbf{A}_{\text {com }}$ of aperiodic monoids with commuting idempotents. In [3], Jackson found the first two examples of limit monoid varieties $\mathbf{L}$ and $\mathbf{M}$. It turned out that $\mathbf{L}$ and $\mathbf{M}$ lie in $\mathbf{A}_{\text {com }}$. Lee established that only $\mathbf{L}$ and $\mathbf{M}$ are limit varieties within several classes of monoid varieties $[6,7]$. In particular, he proved in [7] the uniqueness of the limit varieties $\mathbf{L}$ and $\mathbf{M}$ in an important subclass of $\mathbf{A}_{\text {com }}$, namely, in the class of varieties of aperiodic monoids with central idempotents. Just recently, the third example of a limit variety $\mathbf{J}$ from $\mathbf{A}_{\text {com }}$ was found in [1]. In this article, we completely classify all limit varieties within the class $\mathbf{A}_{\text {com }}$.

In order to formulate the main result of the article, we need some definitions and notation. The free monoid over a countably infinite alphabet is denoted by $F^{1}$. As usual, elements of $F^{1}$ and elements of the alphabet are called words and letters respectively. Words and letters are denoted by small Latin letters. However,

2010 Mathematics Subject Classification. 20M07.

Key words and phrases. Monoid, variety, limit variety, finite basis problem.

The work is supported by the Ministry of Science and Higher Education of the Russian Federation (project FEUZ-2020-0016). 
words unlike letters are written in bold. The following construction was used by Perkins [10] to build the first two examples of non-finitely based finite semigroups. For any set of words $W=\left\{\mathbf{w}_{1}, \mathbf{w}_{2}, \ldots, \mathbf{w}_{k}\right\}$, let $S\left(\mathbf{w}_{1}, \mathbf{w}_{2}, \ldots, \mathbf{w}_{k}\right)$ denote the Rees quotient monoid of $F^{1}$ over the ideal of all words that are not subwords of any word in $W$. The above-mentioned varieties $\mathbf{L}$ and $\mathbf{M}$ are introduced as the varieties generated by the monoid of such a form. Namely, $\mathbf{L}$ and $\mathbf{M}$ denote the varieties generated by the monoids $S(x z x y t y)$ and $S(x y z x t y, x t y z x y)$ respectively.

To introduce the remaining limit varieties from $\mathbf{A}_{\text {com }}$, we need some more definitions and notation. Expressions like to $\mathbf{u} \approx \mathbf{v}$ are used for identities, whereas $\mathbf{u}=\mathbf{v}$ means that the words $\mathbf{u}$ and $\mathbf{v}$ coincide. As usual, the symbol $\mathbb{N}$ stands for the set of all natural numbers. For an arbitrary $n \in \mathbb{N}$, we denote by $S_{n}$ the full symmetric group on the set $\{1,2, \ldots, n\}$. The above-mentioned variety $\mathbf{J}$ is given by the identities

$$
\begin{aligned}
x y x & \approx x y x^{2}, \\
x^{2} y^{2} & \approx y^{2} x^{2}, \\
x y z x y & \approx y x z x y, \\
x y x z t x & \approx x y x z x t x, \\
x z_{1 \pi} z_{2 \pi} \cdots z_{n \pi} x\left(\prod_{i=1}^{n} t_{i} z_{i}\right) & \approx x^{2} z_{1 \pi} z_{2 \pi} \cdots z_{n \pi}\left(\prod_{i=1}^{n} t_{i} z_{i}\right)
\end{aligned}
$$

where $n$ ranges over $\mathbb{N}$ and $\pi$ ranges over $S_{n}$. If $\mathbf{X}$ is a monoid variety then we denote by $\overleftarrow{\mathbf{X}}$ the variety dual to $\mathbf{X}$, i.e., the variety consisting of monoids antiisomorphic to monoids from $\mathbf{X}$.

The main result of the paper is the following

Theorem 1.1. The only limit subvarieties of the class $\mathbf{A}_{\text {com }}$ are $\mathbf{L}, \mathbf{M}, \mathbf{J}$ and $\overleftarrow{\mathbf{J}}$.

Theorem 1.1 shows that there are only four limit varieties within the class $\mathbf{A}_{\text {com. }}$. By contrast, Kozhevnikov proves in [5] that there are continuum many limit varieties of periodic groups. As for the aperiodic monoid varieties, it is known only one example of a limit variety of such a type that does not lie in $\mathbf{A}_{\text {com }}$. This example was provided just recently by Zhang and Luo [17].

The article consists of four sections. Section 2 contains definitions, notation and several known auxiliary results. Section 3 is devoted to the proof of the fact that the monoid variety $\mathbf{O}$ given by the identities (1.2) and

$$
x z x y x t y \approx x z y x t y
$$

is hereditarily finitely based. The proof of Theorem 1.1 is given in Section 4 .

\section{Preliminaries}

A variety of monoids is called completely regular if it consists of completely regular monoids (i.e., unions of groups). Band is a semigroup (monoid) in which every element is an idempotent. If $\mathbf{u}$ and $\mathbf{v}$ are words and $\varepsilon$ is an identity then we will write $\mathbf{u} \underset{\varepsilon}{\approx} \mathbf{v}$ in the case when the identity $\mathbf{u} \approx \mathbf{v}$ follows from $\varepsilon$.

Lemma 2.1. Let $\mathbf{V}$ be a variety of aperiodic monoids that does not contain $S(x y x)$. Suppose that $\mathbf{V}$ is not hereditarily finitely based. Then $\mathbf{V}$ satisfies either (1.1) or

$$
x y x \approx x^{2} y x .
$$


Proof. According to [4, Lemma 5.3], $\mathbf{V}$ satisfies a non-trivial identity of the form $x y x \approx \mathbf{w}$. A completely regular variety of aperiodic monoids is a variety of bands. Since all varieties of band monoids are finitely based [16], $\mathbf{V}$ is non-completely regular. Then $\mathbf{w}=x^{p} y x^{q}$ for some $p$ and $q$ such that $p \geq 2$ or $q \geq 2$ by [2, Proposition 2.2 and Corollary 2.6]. By symmetry, we may assume that $q \geq 2$.

Suppose at first that $p=0$. Then $\mathbf{V}$ satisfies the identity $x^{2} \approx x^{q}$ and, therefore, the identity $x y x \approx y x^{2}$. It follows from [11] that every variety that satisfies the latest identity is finitely based. We obtain a contradiction with the hypothesis.

Suppose now that $p \geq 1$. Then $\mathbf{V}$ satisfies the identity $x^{2} \approx x^{p+q}$ and, therefore, the identity

$$
x^{2} \approx x^{3}
$$

because $\mathbf{V}$ is aperiodic. Then the identities

$$
x y x \approx x^{p} y x^{q} \stackrel{(2.2)}{\approx} x^{p} y x^{q+1} \approx x y x^{2}
$$

hold in $\mathbf{V}$. Thus, (1.1) is satisfied in $\mathbf{V}$.

For an identity system $\Sigma$, we denote by var $\Sigma$ the variety of monoids given by $\Sigma$. Let us fix notation for the following two varieties:

$$
\begin{aligned}
& \mathbf{K}=\operatorname{var}\left\{(1.1),(1.2), x^{2} y \approx x^{2} y x\right\}, \\
& \mathbf{Q}=\operatorname{var}\{(1.1),(1.2),(2.1)\} .
\end{aligned}
$$

The following claim follows from [2, Proposition 6.1] for the variety $\mathbf{K}$ and from $[9$, Condition 4 on page 8$]$ for the variety $\mathbf{Q}$.

Lemma 2.2. The varieties $\mathbf{K}$ and $\mathbf{Q}$ are hereditarily finitely based.

Put

$$
\begin{aligned}
& \mathbf{E}=\operatorname{var}\left\{(1.2),(2.2), y x^{2} \approx x y x\right\}, \\
& \mathbf{F}=\operatorname{var}\left\{(1.1),(1.2),(1.3), x^{2} y \approx x^{2} y x\right\} .
\end{aligned}
$$

To avoid a confusion below, we note that, in $[1,2]$, the variety $\mathbf{E}$ is denoted by $\overleftarrow{\mathbf{E}}$ and $\overleftarrow{\mathbf{E}}$ denotes the variety $\mathbf{E}$, while, in [2], the variety $\mathbf{F}$ is denoted by $\mathbf{F}_{1}$

Lemma 2.3. Let $\mathbf{V}$ be a variety of monoids that satisfies the identities (1.1) and (1.2).

(i) If $\mathbf{E} \nsubseteq \subseteq \mathbf{V}$ then $\mathbf{V} \subseteq \mathbf{K}$.

(ii) If $\mathbf{F} \nsubseteq \mathbf{V}$ then $\mathbf{V} \subseteq \mathbf{Q}$.

Proof. Since (1.1) implies (2.2), $\mathbf{V}$ consists of aperiodic monoids. If $\mathbf{V}$ is completely regular then $\mathbf{V}$ is a variety of bands. Then $\mathbf{V}$ is commutative because it satisfies the identity (1.2). Thus, $\mathbf{V}$ is contained in the variety of all semilattice monoids. In view of [2, Lemma 2.1], $\mathbf{V} \subseteq \mathbf{K} \wedge \mathbf{Q}$. So, we may assume that $\mathbf{V}$ is non-completely regular.

If $\mathbf{E} \nsubseteq \mathbf{V}$ then the identity $x^{2} y \approx x^{2} y x^{2}$ holds in $\mathbf{V}$ by $[2$, the dual to Lemma 4.3]. Then $\mathbf{V}$ satisfies the identities

$$
x^{2} y \approx x^{2} y x^{2} \stackrel{(1.1)}{\approx} x^{2} y x,
$$

whence $\mathbf{V} \subseteq \mathbf{K}$. The claim (i) is proved. 
If $\mathbf{F} \nsubseteq \mathbf{V}$ then arguments from the third paragraph of the proof of Lemma 3.2 of [1] imply that the identity $x y x^{2} \approx x^{2} y x^{2}$ holds in $\mathbf{V}$. Then $\mathbf{V}$ satisfies the identities

$$
x^{2} y x \stackrel{(1.1)}{\approx} x^{2} y x^{2} \approx x y x^{2} \stackrel{(1.1)}{\approx} x y x,
$$

whence $\mathbf{V} \subseteq \mathbf{Q}$. The claim (ii) is proved.

A letter is called simple [multiple] in a word $\mathbf{w}$ if it occurs in $\mathbf{w}$ once [at least twice]. The set of all simple [multiple] letters in a word $\mathbf{w}$ is denoted by $\operatorname{sim}(\mathbf{w})[\mathrm{re}-$ spectively, $\operatorname{mul}(\mathbf{w})]$. The content of a word $\mathbf{w}$, i.e., the set of all letters occurring in $\mathbf{w}$, is denoted by $\operatorname{con}(\mathbf{w})$. The number of occurrences of the letter $x$ in $\mathbf{w}$ is denoted by $\operatorname{occ}_{x}(\mathbf{w})$. For a word $\mathbf{w}$ and letters $x_{1}, x_{2}, \ldots, x_{k} \in \operatorname{con}(\mathbf{w})$, let $\mathbf{w}\left(x_{1}, x_{2}, \ldots, x_{k}\right)$ be the word obtained from $\mathbf{w}$ by deleting all letters except $x_{1}, x_{2}, \ldots, x_{k}$.

Now we are going to define several notions that appear in [1] and [2, Chapter $3]$. Let $\mathbf{w}$ be a word and $\operatorname{sim}(\mathbf{w})=\left\{t_{1}, t_{2}, \ldots, t_{m}\right\}$. We may assume without loss of generality that $\mathbf{w}\left(t_{1}, t_{2}, \ldots, t_{m}\right)=t_{1} t_{2} \cdots t_{m}$. Then $\mathbf{w}=t_{0} \mathbf{w}_{0} t_{1} \mathbf{w}_{1} \cdots t_{m} \mathbf{w}_{m}$ where $\mathbf{w}_{0}, \mathbf{w}_{1}, \ldots, \mathbf{w}_{m}$ are possibly empty words and $t_{0}$ is the empty word. The words $\mathbf{w}_{0}, \mathbf{w}_{1}, \ldots, \mathbf{w}_{m}$ are called blocks of a word $\mathbf{w}$, while $t_{0}, t_{1}, \ldots, t_{m}$ are said to be dividers of $\mathbf{w}$. The representation of the word $\mathbf{w}$ as a product of alternating dividers and blocks starting with the divider $t_{0}$ and ending with the block $\mathbf{w}_{m}$ is called a decomposition of the word $\mathbf{w}$. For a given word $\mathbf{w}$, a letter $x \in \operatorname{con}(\mathbf{w})$ and a natural number $i \leq \operatorname{occ}_{x}(\mathbf{w})$, we denote by $h_{i}(\mathbf{w}, x)$ the right-most divider of $\mathbf{w}$ that precedes the $i$ th occurrence of $x$ in $\mathbf{w}$, and by $t(\mathbf{w}, x)$ the right-most divider of $\mathbf{w}$ that precedes the latest occurrence of $x$ in $\mathbf{w}$.

Lemma 2.4 ( $[1$, Corollary 2.5]). A non-trivial identity $\mathbf{u} \approx \mathbf{v}$ holds in the variety $\mathbf{F} \vee \mathbf{E}$ if and only if the claims

$$
\begin{aligned}
& \operatorname{sim}(\mathbf{u})=\operatorname{sim}(\mathbf{v}) \text { and } \operatorname{mul}(\mathbf{u})=\operatorname{mul}(\mathbf{v}), \\
& h_{1}(\mathbf{u}, x)=h_{1}(\mathbf{v}, x) \text { for all } x \in \operatorname{con}(\mathbf{u}), \\
& h_{2}(\mathbf{u}, x)=h_{2}(\mathbf{v}, x) \text { for all } x \in \operatorname{con}(\mathbf{u}), \\
& t(\mathbf{u}, x)=t(\mathbf{v}, x) \text { for all } x \in \operatorname{con}(\mathbf{u})
\end{aligned}
$$

are true.

The following claim is evident.

Lemma 2.5. Let $\mathbf{u} \approx \mathbf{v}$ be an identity that satisfies the claims (2.3) and (2.4). Suppose that

$$
t_{0} \mathbf{u}_{0} t_{1} \mathbf{u}_{1} \cdots t_{m} \mathbf{u}_{m}
$$

is the decomposition of $\mathbf{u}$. Then the decomposition of $\mathbf{v}$ has the form

$$
t_{0} \mathbf{v}_{0} t_{1} \mathbf{v}_{1} \cdots t_{m} \mathbf{v}_{m}
$$

for some words $\mathbf{v}_{0}, \mathbf{v}_{1}, \ldots, \mathbf{v}_{m}$.

If $\rho$ is an equivalence relation on the free monoid $F^{1}$ then we say that a word $\mathbf{w}$ is a $\rho$-term for a variety $\mathbf{V}$ if $\mathbf{w} \rho \mathbf{w}^{\prime}$ whenever $\mathbf{V}$ satisfies $\mathbf{w} \approx \mathbf{w}^{\prime}$. The following construction from [12] is a generalisation of the construction $S(W)$. Let $\rho$ be a congruence on the free monoid $F^{1}$ and $W$ be a set of words in $F^{1}$ such that the empty word forms a singleton $\rho$-class, $W$ is a union of $\rho$-classes and $W$ is closed under taking subwords. Since $W$ is a union of $\rho$-classes, the ideal $I(W)=F^{1} \backslash W$ is also a union of $\rho$-classes if it is not empty. Let $\varphi_{\rho}$ denote the homomorphism 
LIMIT VARIETIES OF APERIODIC MONOIDS WITH COMMUTING IDEMPOTENTS 5

corresponding to $\rho$. Since $W$ is closed under taking subwords, $\varphi_{\rho}(I(W))$ is an ideal of the quotient monoid $F^{1} / \rho$. We define $S_{\rho}(W)$ as the Rees quotient of $F^{1} / \rho$ over $\varphi_{\rho}(I(W))$.

The following lemma gives us a connection between monoids of the form $S_{\rho}(W)$ and $\rho$-terms for monoid varieties.

Lemma 2.6 ( $\left[12\right.$, Lemma 7.1]). Let $\rho$ be a congruence on the free monoid $F^{1}$ and $W$ be a set of words in $F^{1}$ such that the empty word forms a singleton $\rho$-class, $W$ is a union of $\rho$-classes and $W$ is closed under taking subwords. A monoid variety $\mathbf{V}$ contains $S_{\rho}(W)$ if and only if every word in $W$ is a $\rho$-term for $\mathbf{V}$.

\section{The VARIETy O AND ItS SUbVarieties}

The goal of this section is to prove the following

Proposition 3.1. The variety $\mathbf{O}$ is hereditarily finitely based.

We need the several auxiliary results. The following statement is evident. We will use it below without references.

Lemma 3.1. The identities (1.1) and

$$
x t y z x y \approx x \operatorname{tg} z y x
$$

hold in the variety $\mathbf{O}$.

Lemma 3.2. Let $\mathbf{w}=\mathbf{v}_{1} a \mathbf{v}_{2} a \mathbf{v}_{3}$ where $\mathbf{v}_{1}, \mathbf{v}_{2}$ and $\mathbf{v}_{3}$ are possibly empty words. Suppose that $a \in \operatorname{con}\left(\mathbf{v}_{1}\right)$ and $\operatorname{con}\left(\mathbf{v}_{2}\right) \subseteq \operatorname{mul}(\mathbf{w})$. Then $\mathbf{O}$ satisfies the identity $\mathbf{w} \approx \mathbf{v}_{1} \mathbf{v}_{2} a \mathbf{v}_{3}$.

Proof. Let $\mathbf{v}_{2}=x_{1} x_{2} \cdots x_{n}$ where the letters $x_{1}, x_{2}, \ldots, x_{n}$ are not necessarily different. We will use induction by $n$.

Induction base. Suppose that $n=0$. Here the identity

$$
\mathbf{w}=\mathbf{v}_{1} a^{2} \mathbf{v}_{3} \stackrel{(1.1)}{\approx} \mathbf{v}_{1} a \mathbf{v}_{3}
$$

holds in $\mathbf{O}$ and we are done.

Induction step. Let now $n>0$. If $x_{n} \in \operatorname{con}\left(\mathbf{v}_{1} x_{1} x_{2} \cdots x_{n-1}\right)$ then $\mathbf{O}$ satisfies the identities

$$
\begin{array}{rlrl}
\mathbf{v}_{1} \mathbf{v}_{2} a \mathbf{v}_{3} & =\mathbf{v}_{1} x_{1} x_{2} \cdots x_{n} a \mathbf{v}_{3} & \\
& \approx \mathbf{v}_{1} x_{1} x_{2} \cdots x_{n-1} a x_{n} \mathbf{v}_{3} & & \text { by }(3.1) \\
& \approx \mathbf{v}_{1} a x_{1} x_{2} \cdots x_{n-1} a x_{n} \mathbf{v}_{3} & & \text { by the induction assumption } \\
& \approx \mathbf{v}_{1} a x_{1} x_{2} \cdots x_{n} a \mathbf{v}_{3} & & \text { by }(3.1) \\
& =\mathbf{w} . & &
\end{array}
$$

If $x_{n} \notin \operatorname{con}\left(\mathbf{v}_{1} x_{1} x_{2} \cdots x_{n-1}\right)$ then $x_{n} \in \operatorname{con}\left(\mathbf{v}_{3}\right)$ because $x_{n} \in \operatorname{mul}(\mathbf{w})$. Then the identities

$$
\begin{array}{rlrl}
\mathbf{v}_{1} \mathbf{v}_{2} a \mathbf{v}_{3} & =\mathbf{v}_{1} x_{1} x_{2} \cdots x_{n} a \mathbf{v}_{3} & \\
& \approx \mathbf{v}_{1} x_{1} x_{2} \cdots x_{n-1} a x_{n} a \mathbf{v}_{3} & & \text { by }(1.5) \\
& \approx \mathbf{v}_{1} a x_{1} x_{2} \cdots x_{n-1} a x_{n} a \mathbf{v}_{3} & & \text { by the induction assumption } \\
& \approx \mathbf{v}_{1} a x_{1} x_{2} \cdots x_{n} a \mathbf{v}_{3} & & \text { by }(1.5) \\
& =\mathbf{w} & &
\end{array}
$$


hold in $\mathbf{O}$.

Lemma 3.3. Let $\mathbf{v}_{1}$ and $\mathbf{v}_{2}$ be words. If $\operatorname{con}\left(\mathbf{v}_{2}\right)=\left\{x_{1}, x_{2}, \ldots, x_{n}\right\} \subseteq \operatorname{con}\left(\mathbf{v}_{1}\right)$ then the identity $\mathbf{v}_{1} \mathbf{v}_{2} \approx \mathbf{v}_{1} x_{1} x_{2} \cdots x_{n}$ holds in $\mathbf{O}$.

Proof. The identities

$$
\mathbf{v}_{1} \mathbf{v}_{2} \stackrel{(3.1)}{\approx} \mathbf{v}_{1} x_{1}^{\mathrm{occ}_{x_{1}}\left(\mathbf{v}_{2}\right)} x_{2}^{\mathrm{occ} x_{2}\left(\mathbf{v}_{2}\right)} \cdots x_{n}^{\mathrm{occ} x_{n}\left(\mathbf{v}_{2}\right)} \stackrel{(1.1)}{\approx} \mathbf{v}_{1} x_{1} x_{2} \cdots x_{n}
$$

hold in $\mathbf{O}$.

Let $\mathbf{u}$ and $\mathbf{v}$ be words with decompositions (2.7) and (2.8) respectively. The identity $\mathbf{u} \approx \mathbf{v}$ is not well-balanced at $x$ if $\operatorname{occ}_{x}\left(\mathbf{u}_{i}\right) \neq \operatorname{occ}_{x}\left(\mathbf{v}_{i}\right)$ for some $i$. For brevity, put $\mathbf{O}\{\Sigma\}=\mathbf{O} \wedge \operatorname{var}\{\Sigma\}$ for any identity system $\Sigma$.

Lemma 3.4. Let $\mathbf{u} \approx \mathbf{v}$ be an identity that holds in $\mathbf{F} \vee \mathbf{E}$. Then either

$$
\mathbf{O}\{\mathbf{u} \approx \mathbf{v}\}=\mathbf{O}\left\{\mathbf{u}^{\prime} \approx \mathbf{v}^{\prime}\right\} \text { or } \mathbf{O}\{\mathbf{u} \approx \mathbf{v}\}=\mathbf{O}\left\{\mathbf{u}^{\prime} \approx \mathbf{v}^{\prime},(1.4)\right\}
$$

for some well-balanced identity $\mathbf{u}^{\prime} \approx \mathbf{v}^{\prime}$.

Proof. If $\mathbf{u} \approx \mathbf{v}$ is well-balanced then we are done. Suppose that $\mathbf{u} \approx \mathbf{v}$ is not well-balanced. By induction, we may assume that this identity is not well-balanced at precisely one letter $x$. In view of Lemma 2.4, the claims (2.4)-(2.6) are true. Let (2.7) be the decomposition of $\mathbf{u}$. Then the decomposition of $\mathbf{v}$ has the form (2.8) by Lemma 2.5. By induction, we may assume that there is $k$ such that $\operatorname{occ}_{x}\left(\mathbf{u}_{k}\right) \neq$ $\operatorname{occ}_{x}\left(\mathbf{v}_{k}\right)$ but $\operatorname{occ}_{x}\left(\mathbf{u}_{i}\right)=\operatorname{occ}_{x}\left(\mathbf{v}_{i}\right)$ for any $i \neq k$. We may assume without any loss that $\operatorname{occ}_{x}\left(\mathbf{u}_{k}\right)<\operatorname{occ}_{x}\left(\mathbf{v}_{k}\right)$. Put

$$
\mathbf{u}^{\prime}=\prod_{i=0}^{k-1}\left(\mathbf{u}_{i} t_{i+1}\right), \mathbf{u}^{\prime \prime}=\prod_{i=k+1}^{m}\left(t_{i} \mathbf{u}_{i}\right), \mathbf{v}^{\prime}=\prod_{i=0}^{k-1}\left(\mathbf{v}_{i} t_{i+1}\right), \mathbf{v}^{\prime \prime}=\prod_{i=k+1}^{m}\left(t_{i} \mathbf{v}_{i}\right) .
$$

Suppose that $x \in \operatorname{con}\left(\mathbf{u}_{k}\right)$. It follows that $\operatorname{occ}_{x}\left(\mathbf{v}_{k}\right) \geq 2$. Therefore, the first and the second occurrences of $x$ in $\mathbf{v}$ lie in the subword $\mathbf{v}^{\prime} \mathbf{v}_{k}$. In view of the claims (2.4) and (2.5), the first and the second occurrences of $x$ in $\mathbf{u}$ lie in the subword $\mathbf{u}^{\prime} \mathbf{u}_{k}$. Then there are subwords $\mathbf{u}_{k}^{\prime}$ and $\mathbf{u}_{k}^{\prime \prime}$ of $\mathbf{u}_{k}$ such that $\mathbf{u}_{k}=\mathbf{u}_{k}^{\prime} x \mathbf{u}_{k}^{\prime \prime}$ and $x \in \operatorname{con}\left(\mathbf{u}^{\prime} \mathbf{u}_{k}^{\prime}\right)$. Put

$$
\mathbf{p}=\mathbf{u}_{k}^{\prime} x^{\mathrm{occ}_{x}\left(\mathbf{v}_{k}\right)-\mathrm{occ}_{x}\left(\mathbf{u}_{k}\right)+1} \mathbf{u}_{k}^{\prime \prime}
$$

The identity $\mathbf{u} \stackrel{(1.1)}{\approx} \mathbf{u}^{\prime} \mathbf{p} \mathbf{u}^{\prime \prime}$ holds in $\mathbf{O}$, whence $\mathbf{O}\{\mathbf{u} \approx \mathbf{v}\}=\mathbf{O}\left\{\mathbf{u}^{\prime} \mathbf{p} \mathbf{u}^{\prime \prime} \approx \mathbf{v}\right\}$. It remains to note that the identity $\mathbf{u}^{\prime} \mathbf{p} \mathbf{u}^{\prime \prime} \approx \mathbf{v}$ is well-balanced.

Suppose now that $x \notin \operatorname{con}\left(\mathbf{u}_{k}\right)$. In view of [8, Lemma 5.1], $\mathbf{Q} \nsubseteq \mathbf{O}\{\mathbf{u} \approx \mathbf{v}\}$. This fact and [8, Lemma 5.3] imply that $\mathbf{O}\{\mathbf{u} \approx \mathbf{v}\}$ satisfies the identity

$$
x^{2} y z x^{2} \approx x^{2} y x z x^{2} .
$$

Then the identities

$$
x y x z t x \stackrel{(1.1)}{\approx} x y x^{2} z t x^{2} \stackrel{(3.2)}{\approx} x y x^{2} z x t x^{2} \stackrel{(1.1)}{\approx} x y x z x t x
$$

hold in the variety $\mathbf{O}\{\mathbf{u} \approx \mathbf{v}\}$. We see that this variety satisfies the identity (1.4). The second occurrence of $x$ in $\mathbf{v}$ does not lie in the block $\mathbf{v}_{k}$ by the claim (2.5). If $t_{j}=h_{2}(\mathbf{v}, x)$ for some $j>k$ then we obtain a contradiction with the claim (2.4) and the fact that $x \in \operatorname{con}\left(\mathbf{v}_{k}\right) \backslash \operatorname{con}\left(\mathbf{u}_{k}\right)$. So, the second occurrence of $x$ in $\mathbf{v}$ lies in the subword $\mathbf{v}^{\prime}$. Taking into account the claims (2.4) and (2.5) again, we get that the first and the second occurrences of $x$ in $\mathbf{u}$ lie in the subword $\mathbf{u}^{\prime}$. 
Further, the fact that $x \in \operatorname{con}\left(\mathbf{v}_{k}\right) \backslash \operatorname{con}\left(\mathbf{u}_{k}\right)$ and the claim (2.6) imply that the latest occurrences of $x$ in $\mathbf{u}$ and $\mathbf{v}$ lie in the subwords $\mathbf{u}^{\prime \prime}$ and $\mathbf{v}^{\prime \prime}$ respectively. Put $\mathbf{q}=\mathbf{u}_{k} x^{\mathrm{occ}_{x}\left(\mathbf{v}_{k}\right)}$. Then the identity $\mathbf{u} \stackrel{(1.4)}{\approx} \mathbf{u}^{\prime} \mathbf{q} \mathbf{u}^{\prime \prime}$ holds in the variety $\mathbf{O}\{(1.4)\}$, whence $\mathbf{O}\{\mathbf{u} \approx \mathbf{v}\}=\mathbf{O}\left\{\mathbf{u}^{\prime} \mathbf{q} \mathbf{u}^{\prime \prime} \approx \mathbf{v},(1.4)\right\}$. It remains to note that the identity $\mathbf{u}^{\prime} \mathbf{q u} \mathbf{u}^{\prime \prime} \approx \mathbf{v}$ is well-balanced.

The identity $\mathbf{u} \approx \mathbf{v}$ is said to be 1-invertible if $\mathbf{u}=\mathbf{w}^{\prime} x y \mathbf{w}^{\prime \prime}$ and $\mathbf{v}=\mathbf{w}^{\prime} y x \mathbf{w}^{\prime \prime}$ for some possibly empty words $\mathbf{w}^{\prime}, \mathbf{w}^{\prime \prime}$ and some letters $x, y \in \operatorname{con}\left(\mathbf{w}^{\prime} \mathbf{w}^{\prime \prime}\right)$. Let now $n>1$. The identity $\mathbf{u} \approx \mathbf{v}$ is said to be $n$-invertible if there exists a sequence of words

$$
\mathbf{u}=\mathbf{w}_{0}, \mathbf{w}_{1}, \ldots, \mathbf{w}_{n}=\mathbf{v}
$$

such that the identity $\mathbf{w}_{j} \approx \mathbf{w}_{j+1}$ is 1 -invertible for any $j \in\{0,1, \ldots, n-1\}$ and $n$ is the least number with such a property. For convenience, the trivial identity is called 0-invertible.

Lemma 3.5. Let $\mathbf{u} \approx \mathbf{v}$ be a well-balanced identity. Then the variety $\mathbf{O}\{\mathbf{u} \approx \mathbf{v}\}$ can be defined by the identities (1.2) and (1.5) together with some of the following identities: (1.3),

$$
\begin{aligned}
y x^{2} t x y & \approx x y x t x y, \\
x^{2} y t x y & \approx x y x t x y, \\
\alpha_{n}: x y \prod_{i=1}^{n+1}\left(t_{i} \mathbf{e}_{i}\right) & \approx y x \prod_{i=1}^{n+1}\left(t_{i} \mathbf{e}_{i}\right), \\
\beta_{n}: y x^{2} \prod_{i=2}^{n+1}\left(t_{i} \mathbf{e}_{i}\right) & \approx x y x \prod_{i=1}^{n}\left(t_{i} \mathbf{e}_{i}\right), \\
\gamma_{n}: x^{2} y \prod_{i=1}^{n+1}\left(t_{i} \mathbf{e}_{i}\right) & \approx x y x \prod_{i=1}^{n+1}\left(t_{i} \mathbf{e}_{i}\right), \\
\gamma_{n}^{\prime}: x^{2} y \prod_{i=2}^{n+1}\left(t_{i} \mathbf{e}_{i}\right) & \approx x y x \prod_{i=2}^{n+1}\left(t_{i} \mathbf{e}_{i}\right),
\end{aligned}
$$

where $n \in \mathbb{N}$ and

$$
\mathbf{e}_{i}= \begin{cases}x & \text { if } i \text { is odd } \\ y & \text { if } i \text { is even }\end{cases}
$$

Proof. Put

$$
\Phi=\left\{(1.3),(3.3),(3.4), \alpha_{n}, \beta_{n}, \gamma_{n}, \gamma_{n}^{\prime} \mid n \in \mathbb{N}\right\} .
$$

Since the identity $\mathbf{u} \approx \mathbf{v}$ is well-balanced, it is $n$-invertible for some $n \geq 0$. We will use induction by $n$.

Induction base. Suppose that $n=0$. Here $\mathbf{u}=\mathbf{v}$, whence $\mathbf{O}\{\mathbf{u} \approx \mathbf{v}\}=\mathbf{O}\{\emptyset\}$.

Induction step. Let now $n>0$. Let (2.7) be the decomposition of $\mathbf{u}$. Then the decomposition of $\mathbf{v}$ has the form (2.8) because $\mathbf{u} \approx \mathbf{v}$ is well-balanced. There is $0 \leq i \leq m$ such that $\mathbf{u}_{i} \neq \mathbf{v}_{i}$. Let $\mathbf{p}$ be the greatest common prefix of $\mathbf{u}_{i}$ and $\mathbf{v}_{i}$. Suppose that $\mathbf{u}_{i}=\mathbf{p} x \mathbf{u}_{i}^{\prime}$ for some letter $x$ and some word $\mathbf{u}_{i}^{\prime}$. Since $\mathbf{u} \approx \mathbf{v}$ 
is well-balanced, there are words $\mathbf{a}, \mathbf{b}$ and a letter $y$ such that $\mathbf{v}_{i}=\mathbf{p a} y x \mathbf{b}$ and $x \notin \operatorname{con}(\mathbf{a} y)$. We note also that $y \in \operatorname{con}\left(\mathbf{u}_{i}^{\prime}\right)$ because $\mathbf{u} \approx \mathbf{v}$ is well-balanced. Put

$$
\mathbf{v}^{\prime}=\prod_{j=0}^{i-1}\left(\mathbf{v}_{j} t_{j+1}\right) \mathbf{p}, \mathbf{v}^{\prime \prime}=\prod_{j=i+1}^{m}\left(t_{j} \mathbf{v}_{j}\right) \text { and } \mathbf{w}=\mathbf{v}^{\prime} \mathbf{a} x y \mathbf{b} \mathbf{v}^{\prime \prime} .
$$

We are going to verify that

$$
\text { either } \mathbf{O}\{\mathbf{u} \approx \mathbf{v}\}=\mathbf{O}\{\mathbf{u} \approx \mathbf{w}\} \text { or } \mathbf{O}\{\mathbf{u} \approx \mathbf{v}\}=\mathbf{O}\{\mathbf{u} \approx \mathbf{w}, \sigma\}
$$

for some $\sigma \in \Phi$.

Suppose that $x, y \in \operatorname{con}\left(\mathbf{v}^{\prime} \mathbf{a b}\right)$. If $x, y \in \operatorname{con}(\mathbf{b})$ then the identities

$$
\begin{aligned}
\mathbf{w} & =\mathbf{v}^{\prime} \mathbf{a} x y \mathbf{b} \mathbf{v}^{\prime \prime} & & \\
& \approx \mathbf{v}^{\prime} \mathbf{a} x^{2} y^{2} \mathbf{b} \mathbf{v}^{\prime \prime} & & \text { by Lemma } 3.2 \\
& \approx \mathbf{v}^{\prime} \mathbf{a} y^{2} x^{2} \mathbf{b} \mathbf{v}^{\prime \prime} & & \text { by }(1.2) \\
& \approx \mathbf{v}^{\prime} \mathbf{a} y x \mathbf{b} \mathbf{v}^{\prime \prime \prime} & & \text { by Lemma } 3.2 \\
& =\mathbf{v} & &
\end{aligned}
$$

hold in $\mathbf{O}$, whence $\mathbf{O}\{\mathbf{u} \approx \mathbf{v}\}=\mathbf{O}\{\mathbf{u} \approx \mathbf{w}\}$. So, we may assume that either $x \notin \operatorname{con}(\mathbf{b})$ or $y \notin \operatorname{con}(\mathbf{b})$. By symmetry, it suffices to consider the case $y \notin \operatorname{con}(\mathbf{b})$. Then $y \in \operatorname{con}\left(\mathbf{v}^{\prime} \mathbf{a}\right)$. If $x \in \operatorname{con}(\mathbf{b})$ then the identities

$$
\begin{aligned}
\mathbf{w} & =\mathbf{v}^{\prime} \mathbf{a} x y \mathbf{b} \mathbf{v}^{\prime \prime} & & \\
& \approx \mathbf{v}^{\prime} \mathbf{a} x^{2} y \mathbf{b} \mathbf{v}^{\prime \prime} & & \text { by Lemma } 3.2 \\
& \approx \mathbf{v}^{\prime} \mathbf{a} x^{2} y^{2} \mathbf{b} \mathbf{v}^{\prime \prime} & & \text { by }(1.1) \\
& \approx \mathbf{v}^{\prime} \mathbf{a} y^{2} x^{2} \mathbf{b} \mathbf{v}^{\prime \prime} & & \text { by }(1.2) \\
& \approx \mathbf{v}^{\prime} \mathbf{a} y x^{2} \mathbf{b} \mathbf{v v}^{\prime \prime} & & \text { by }(1.1) \\
& \approx \mathbf{v}^{\prime} \mathbf{a} y x \mathbf{b} \mathbf{v v}^{\prime \prime \prime} & & \text { by Lemma } 3.2 \\
& =\mathbf{v} & &
\end{aligned}
$$

hold in $\mathbf{O}$. Therefore, $\mathbf{O}\{\mathbf{u} \approx \mathbf{v}\}=\mathbf{O}\{\mathbf{u} \approx \mathbf{w}\}$. Finally, if $x \notin \operatorname{con}(\mathbf{b})$ then $x \in \operatorname{con}\left(\mathbf{v}^{\prime} \mathbf{a}\right)$ and the identities

$$
\mathbf{w}=\mathbf{v}^{\prime} \mathbf{a} x y \mathbf{b} \mathbf{v}^{\prime \prime} \stackrel{(3.1)}{\approx} \mathbf{v}^{\prime} \mathbf{a} y x \mathbf{b} \mathbf{v}^{\prime \prime}=\mathbf{v}
$$

hold in $\mathbf{O}$, whence $\mathbf{O}\{\mathbf{u} \approx \mathbf{v}\}=\mathbf{O}\{\mathbf{u} \approx \mathbf{w}\}$ again. Thus, it remains to consider the case when either $x \notin \operatorname{con}\left(\mathbf{v}^{\prime} \mathbf{a b}\right)$ or $y \notin \operatorname{con}\left(\mathbf{v}^{\prime} \mathbf{a b}\right)$. By symmetry, we may assume without loss of generality that $y \notin \operatorname{con}\left(\mathbf{v}^{\prime} \mathbf{a b}\right)$. Then $y \in \operatorname{con}\left(\mathbf{v}^{\prime \prime}\right)$ because $y \in \operatorname{mul}(\mathbf{v})$.

Suppose that $x \in \operatorname{con}\left(\mathbf{v}^{\prime} \mathbf{a}\right) \cap \operatorname{con}(\mathbf{b})$. Then the identities

$$
\begin{aligned}
& \mathbf{w}=\mathbf{v}^{\prime} \mathbf{a} x y \mathbf{b v}^{\prime \prime} \\
& \approx \mathbf{v}^{\prime} \mathbf{a} y \mathbf{b} \mathbf{v}^{\prime \prime} \quad \text { by Lemma } 3.2 \\
& \approx \mathbf{v}^{\prime} \mathbf{a} y x \mathbf{b v}^{\prime \prime \prime} \quad \text { by Lemma } 3.2 \\
& =\mathbf{v}
\end{aligned}
$$


LIMIT VARIETIES OF APERIODIC MONOIDS WITH COMMUTING IDEMPOTENTS 9

hold in $\mathbf{O}$, whence $\mathbf{O}\{\mathbf{u} \approx \mathbf{v}\}=\mathbf{O}\{\mathbf{u} \approx \mathbf{w}\}$. So, we may assume that either $x \notin \operatorname{con}\left(\mathbf{v}^{\prime} \mathbf{a}\right)$ or $x \notin \operatorname{con}(\mathbf{b})$. Put

$$
\mathbf{w}_{j}=t_{i+1} \prod_{s=i+2}^{j}\left(\mathbf{v}_{s-1} t_{s}\right) \text { and } \mathbf{w}_{j}^{\prime}=\prod_{s=j+1}^{m}\left(t_{s} \mathbf{v}_{s}\right)
$$

for any $j>i$.

Case 1: $x \notin \operatorname{con}\left(\mathbf{v}^{\prime} \mathbf{a b}\right)$. Then $x \in \operatorname{con}\left(\mathbf{v}^{\prime \prime}\right)$ because $x \in \operatorname{mul}(\mathbf{v})$.

Subcase 1.1: $x, y \in \operatorname{con}\left(\mathbf{v}_{k}\right)$ for some $k>i$. Then, since $\mathbf{u} \approx \mathbf{v}$ is well-balanced, $\mathbf{u}\left(x, y, t_{i+1}\right)=x y t_{i+1} \mathbf{p}$ and $\mathbf{v}\left(x, y, t_{i+1}\right)=y x t_{i+1} \mathbf{q}$ where $\operatorname{con}(\mathbf{p})=\operatorname{con}(\mathbf{q})=$ $\{x, y\}$. According to Lemma 3.3,

$$
\mathbf{O}\left\{\mathbf{u}\left(x, y, t_{i+1}\right) \approx \mathbf{v}\left(x, y, t_{i+1}\right)\right\}=\mathbf{O}\{(1.3)\} .
$$

Therefore, $\mathbf{O}\{\mathbf{u} \approx \mathbf{v}\}$ satisfies (1.3). On the other hand, the identities

$$
\begin{aligned}
\mathbf{w} & =\mathbf{v}^{\prime} \mathbf{a} x y \mathbf{b} \mathbf{w}_{k} \mathbf{v}_{k} \mathbf{w}_{k}^{\prime} & & \\
& \approx \mathbf{v}^{\prime} \mathbf{a} x y \mathbf{b} \mathbf{w}_{k} x y \mathbf{v}_{k} \mathbf{w}_{k}^{\prime} & & \text { by Lemma } 3.2 \\
& \approx \mathbf{v}^{\prime} \mathbf{a} y x \mathbf{b} \mathbf{w}_{k} x y \mathbf{v}_{k} \mathbf{w}_{k}^{\prime} & & \text { by }(1.3) \\
& \approx \mathbf{v}^{\prime} \mathbf{a} y x \mathbf{b} \mathbf{w}_{k} \mathbf{v}_{k} \mathbf{w}_{k}^{\prime} & & \text { by Lemma } 3.2 \\
& =\mathbf{v} & &
\end{aligned}
$$

hold in $\mathbf{O}\{(1.3)\}$, whence $\mathbf{O}\{\mathbf{u} \approx \mathbf{v}\}=\mathbf{O}\{\mathbf{u} \approx \mathbf{w},(1.3)\}$.

Subcase 1.2: $\left|\operatorname{con}\left(\mathbf{u}_{j}\right) \cap\{x, y\}\right| \leq 1$ for any $j>i$. Then we may assume without any loss that there exists a subsequence $k_{1}, k_{2}, \ldots, k_{r}=m+1$ of $i+1, i+2, \ldots, m+1$ such that

(3.6) if $s$ is odd and $s \leq r-1$ then $\operatorname{con}\left(\mathbf{u}_{k_{s}} \mathbf{u}_{k_{s}+1} \cdots \mathbf{u}_{k_{s+1}-1}\right) \cap\{x, y\}=\{x\}$;

$$
\operatorname{con}\left(\mathbf{u}_{i+1} \mathbf{u}_{i+2} \cdots \mathbf{u}_{k_{1}-1}\right) \cap\{x, y\}=\emptyset ;
$$

if $s$ is even and $s \leq r-1$ then $\operatorname{con}\left(\mathbf{u}_{k_{s}} \mathbf{u}_{k_{s}+1} \cdots \mathbf{u}_{k_{s+1}-1}\right) \cap\{x, y\}=\{y\}$.

Clearly, $r>2$ because the letters $x$ and $y$ are multiple in $\mathbf{u}$ and $\mathbf{v}$. Then, since $\mathbf{u} \approx \mathbf{v}$ is well-balanced,

$$
\begin{aligned}
& \mathbf{u}\left(x, y, t_{k_{1}}, t_{k_{2}}, \ldots, t_{k_{r-1}}\right)=x y \prod_{j=1}^{r-1}\left(t_{k_{j}} \mathbf{f}_{j}\right), \\
& \mathbf{v}\left(x, y, t_{k_{1}}, t_{k_{2}}, \ldots, t_{k_{r-1}}\right)=y x \prod_{j=1}^{r-1}\left(t_{k_{j}} \mathbf{f}_{j}\right)
\end{aligned}
$$

where

$$
\mathbf{f}_{j}= \begin{cases}x^{a_{j}} & \text { if } j \text { is odd } \\ y^{a_{j}} & \text { if } j \text { is even }\end{cases}
$$

for some $a_{j} \in \mathbb{N}$ and $j=1,2, \ldots, r-1$. In view of Lemma 3.3,

$$
\mathbf{O}\left\{\mathbf{u}\left(x, y, t_{k_{1}}, t_{k_{2}}, \ldots, t_{k_{r-1}}\right) \approx \mathbf{v}\left(x, y, t_{k_{1}}, t_{k_{2}}, \ldots, t_{k_{r-1}}\right)\right\}=\mathbf{O}\left\{\alpha_{r-2}\right\} .
$$

Therefore, $\mathbf{O}\{\mathbf{u} \approx \mathbf{v}\}$ satisfies the identity $\alpha_{r-2}$. On the other hand, the identities

$$
\mathbf{w}=\mathbf{v}^{\prime} \mathbf{a} x y \mathbf{b} \mathbf{v}^{\prime \prime} \stackrel{\alpha_{r}-2}{\approx} \mathbf{v}^{\prime} \mathbf{a} y x \mathbf{b} \mathbf{v}^{\prime \prime}=\mathbf{v}
$$

hold in $\mathbf{O}\left\{\alpha_{r-2}\right\}$, whence $\mathbf{O}\{\mathbf{u} \approx \mathbf{v}\}=\mathbf{O}\left\{\mathbf{u} \approx \mathbf{w}, \alpha_{r-2}\right\}$. 
Case 2: $x \notin \operatorname{con}\left(\mathbf{v}^{\prime} \mathbf{a}\right)$ but $x \in \operatorname{con}(\mathbf{b})$.

Subcase 2.1: $x, y \in \operatorname{con}\left(\mathbf{v}_{k}\right)$ for some $k>i$. Since $\mathbf{u} \approx \mathbf{v}$ is well-balanced, substituting $x t_{i+1}$ for $t_{i+1}$ and 1 for all letters occurring in $\mathbf{u} \approx \mathbf{v}$ except $x, y, t_{i+1}$, we obtain the identity

$$
x^{c} y x^{d} t_{i+1} \mathbf{p} \approx y x^{e} t_{i+1} \mathbf{q}
$$

where $c, d \geq 1, e \geq 2$ and $\operatorname{con}(\mathbf{p})=\operatorname{con}(\mathbf{q})=\{x, y\}$. Lemma 3.2 implies that

$$
\mathbf{O}\left\{x^{c} y x^{d} t_{i+1} \mathbf{p} \approx y x^{e} t_{i+1} \mathbf{q}\right\}=\mathbf{O}\left\{x y x t_{i+1} \mathbf{p} \approx y x^{2} t_{i+1} \mathbf{q}\right\} .
$$

According to Lemma 3.3,

$$
\mathbf{O}\left\{x y x t_{i+1} \mathbf{p} \approx y x^{2} t_{i+1} \mathbf{q}\right\}=\mathbf{O}\{(3.3)\} .
$$

Therefore, $\mathbf{O}\{\mathbf{u} \approx \mathbf{v}\}$ satisfies (3.3). On the other hand, the identities

$$
\begin{aligned}
\mathbf{w} & =\mathbf{v}^{\prime} \mathbf{a} x y \mathbf{b} \mathbf{w}_{k} \mathbf{v}_{k} \mathbf{w}_{k}^{\prime} & & \\
& \approx \mathbf{v}^{\prime} \mathbf{a} x y x \mathbf{b} \mathbf{w}_{k} x y \mathbf{v}_{k} \mathbf{w}_{k}^{\prime} & & \text { by Lemma } 3.2 \\
& \approx \mathbf{v}^{\prime} \mathbf{a} y x^{2} \mathbf{b} \mathbf{w}_{k} x y \mathbf{v}_{k} \mathbf{w}_{k}^{\prime} & & \text { by }(3.3) \\
& \approx \mathbf{v}^{\prime} \mathbf{a} y x \mathbf{b} \mathbf{w}_{k} \mathbf{v}_{k} \mathbf{w}_{k}^{\prime} & & \text { by Lemma } 3.2 \\
& =\mathbf{v} & &
\end{aligned}
$$

hold in $\mathbf{O}\{(3.3)\}$, whence $\mathbf{O}\{\mathbf{u} \approx \mathbf{v}\}=\mathbf{O}\{\mathbf{u} \approx \mathbf{w},(3.3)\}$.

Subcase 2.2: $\left|\operatorname{con}\left(\mathbf{u}_{j}\right) \cap\{x, y\}\right| \leq 1$ for any $j>i$. Then there exists a subsequence $k_{1}, k_{2}, \ldots, k_{r}=m+1$ of $i+1, i+2, \ldots, m+1$ such that

$$
\operatorname{con}\left(\mathbf{u}_{i+1} \mathbf{u}_{i+2} \cdots \mathbf{u}_{k_{1}-1}\right) \cap\{x, y\} \subseteq\{x\} ;
$$

(3.9) if $s$ is even and $s \leq r-1$ then $\operatorname{con}\left(\mathbf{u}_{k_{s}} \mathbf{u}_{k_{s}+1} \cdots \mathbf{u}_{k_{s+1}-1}\right) \cap\{x, y\}=\{x\}$;

(3.10) if $s$ is odd and $s \leq r-1$ then $\operatorname{con}\left(\mathbf{u}_{k_{s}} \mathbf{u}_{k_{s}+1} \cdots \mathbf{u}_{k_{s+1}-1}\right) \cap\{x, y\}=\{y\}$.

Clearly, $r>1$ because $y$ is multiple in $\mathbf{u}$ and $\mathbf{v}$. Since $\mathbf{u} \approx \mathbf{v}$ is well-balanced, substituting $x t_{k_{1}}$ for $t_{k_{1}}$ and 1 for all letters occurring in $\mathbf{u} \approx \mathbf{v}$ except $x, y$, $t_{k_{1}}, t_{k_{2}}, \ldots, t_{k_{r-1}}$, we obtain the identity

$$
x^{c} y x^{d} \prod_{j=1}^{r-1}\left(t_{k_{j}} \mathbf{f}_{j}\right) \approx y x^{e} \prod_{j=1}^{r-1}\left(t_{k_{j}} \mathbf{f}_{j}\right)
$$

where $c, d \geq 1, e \geq 2$ and

$$
\mathbf{f}_{j}= \begin{cases}y^{a_{j}} & \text { if } j \text { is odd } \\ x^{a_{j}} & \text { if } j \text { is even }\end{cases}
$$

for some $a_{j} \in \mathbb{N}$ and $j=1,2, \ldots, r-1$. Lemma 3.2 implies that

$$
\mathbf{O}\{(3.11)\}=\mathbf{O}\left\{x y x \prod_{j=1}^{r-1}\left(t_{k_{j}} \mathbf{f}_{j}\right) \approx y x^{2} \prod_{j=1}^{r-1}\left(t_{k_{j}} \mathbf{f}_{j}\right)\right\} .
$$

In view of Lemma 3.3,

$$
\mathbf{O}\left\{x y x \prod_{j=1}^{r-1}\left(t_{k_{j}} \mathbf{f}_{j}\right) \approx y x^{2} \prod_{j=1}^{r-1}\left(t_{k_{j}} \mathbf{f}_{j}\right)\right\}=\mathbf{O}\left\{\beta_{r-1}\right\} .
$$


Therefore, $\mathbf{O}\{\mathbf{u} \approx \mathbf{v}\}$ satisfies $\beta_{r-1}$. On the other hand, the identities

$$
\begin{aligned}
\mathbf{w} & =\mathbf{v}^{\prime} \mathbf{a} x y \mathbf{b} \mathbf{v}^{\prime \prime} & & \\
& \approx \mathbf{v}^{\prime} \mathbf{a} x y x \mathbf{b} \mathbf{v}^{\prime \prime} & & \text { by Lemma } 3.2 \\
& \approx \mathbf{v}^{\prime} \mathbf{a} y x^{2} \mathbf{b} \mathbf{v}^{\prime \prime \prime} & & \text { by the identity } \beta_{r-1} \\
& \approx \mathbf{v}^{\prime} \mathbf{a} y x \mathbf{b} \mathbf{v v}^{\prime \prime \prime} & & \text { by Lemma } 3.2 \\
& =\mathbf{v} & &
\end{aligned}
$$

hold in $\mathbf{O}\left\{\beta_{r-1}\right\}$, whence $\mathbf{O}\{\mathbf{u} \approx \mathbf{v}\}=\mathbf{O}\left\{\mathbf{u} \approx \mathbf{w}, \beta_{r-1}\right\}$.

Case 3: $x \in \operatorname{con}\left(\mathbf{v}^{\prime} \mathbf{a}\right)$ but $x \notin \operatorname{con}(\mathbf{b})$. Then $x \notin \operatorname{con}\left(\mathbf{u}_{i}^{\prime}\right)$ because $x \notin \operatorname{con}(\mathbf{a})$ and $\operatorname{con}\left(\mathbf{u}_{i}\right)=\operatorname{con}\left(\mathbf{v}_{i}\right)$.

Subcase 3.1: $x, y \in \operatorname{con}\left(\mathbf{v}_{k}\right)$ for some $k>i$. Then, since $\mathbf{u} \approx \mathbf{v}$ is well-balanced,

$$
\mathbf{u}\left(x, y, t_{i+1}\right)=x^{c+1} y t_{i+1} \mathbf{p} \text { and } \mathbf{v}\left(x, y, t_{i+1}\right)=x^{c} y x t_{i+1} \mathbf{q}
$$

where $c \in \mathbb{N}$ and $\operatorname{con}(\mathbf{p})=\operatorname{con}(\mathbf{q})=\{x, y\}$. The identities

$$
\mathbf{u}\left(x, y, t_{i+1}\right) \stackrel{(1.1)}{\approx} x^{2} y t_{i+1} \mathbf{p} \text { and } \mathbf{v}\left(x, y, t_{i+1}\right) \stackrel{(1.5)}{\approx} x y x t_{i+1} \mathbf{q}
$$

hold in O. According to Lemma 3.3,

$$
\mathbf{O}\left\{x^{2} y t_{i+1} \mathbf{p} \approx x y x t_{i+1} \mathbf{q}\right\}=\mathbf{O}\{(3.4)\} .
$$

Therefore, $\mathbf{O}\{\mathbf{u} \approx \mathbf{v}\}$ satisfies (3.4). On the other hand, the identities

$$
\begin{aligned}
\mathbf{w} & =\mathbf{v}^{\prime} \mathbf{a} x y \mathbf{b} \mathbf{w}_{k} \mathbf{v}_{k} \mathbf{w}_{k}^{\prime} & & \\
& \approx \mathbf{v}^{\prime} \mathbf{a} x^{2} y \mathbf{b} \mathbf{w}_{k} x y \mathbf{v}_{k} \mathbf{w}_{k}^{\prime} & & \text { by Lemma } 3.2 \\
& \approx \mathbf{v}^{\prime} \mathbf{a} x y x \mathbf{b} \mathbf{w}_{k} x y \mathbf{v}_{k} \mathbf{w}_{k}^{\prime} & & \text { by }(3.4) \\
& \approx \mathbf{v}^{\prime} \mathbf{a} y x \mathbf{b} \mathbf{w}_{k} \mathbf{v}_{k} \mathbf{w}_{k}^{\prime} & & \text { by Lemma } 3.2 \\
& =\mathbf{v} & &
\end{aligned}
$$

hold in $\mathbf{O}\{(3.4)\}$, whence $\mathbf{O}\{\mathbf{u} \approx \mathbf{v}\}=\mathbf{O}\{\mathbf{u} \approx \mathbf{w},(3.4)\}$.

Subcase $3.2:\left|\operatorname{con}\left(\mathbf{u}_{j}\right) \cap\{x, y\}\right| \leq 1$ for any $j>i$. Then there exists a subsequence $k_{1}, k_{2}, \ldots, k_{r}=m+1$ of $i+1, i+2, \ldots, m+1$ such that the claim (3.5) is true and either the claims (3.6) and (3.7) are true or the claims (3.9) and (3.10) are true. Then

$$
\begin{aligned}
& \mathbf{u}\left(x, y, t_{k_{1}}, t_{k_{2}}, \ldots, t_{k_{r-1}}\right)=x^{c+1} y \prod_{j=1}^{r-1}\left(t_{k_{j}} \mathbf{f}_{j}\right), \\
& \mathbf{v}\left(x, y, t_{k_{1}}, t_{k_{2}}, \ldots, t_{k_{r-1}}\right)=x^{c} y x \prod_{j=1}^{r-1}\left(t_{k_{j}} \mathbf{f}_{j}\right)
\end{aligned}
$$

where $c \in \mathbb{N}$ and either (3.8) or (3.12) holds. The identities

$$
\begin{aligned}
& \mathbf{u}\left(x, y, t_{k_{1}}, t_{k_{2}}, \ldots, t_{k_{r-1}}\right) \stackrel{(1.1)}{\approx} x^{2} y \prod_{j=1}^{r-1}\left(t_{k_{j}} \mathbf{f}_{j}\right), \\
& \mathbf{v}\left(x, y, t_{k_{1}}, t_{k_{2}}, \ldots, t_{k_{r-1}}\right) \stackrel{(1.5)}{\approx} x y x \prod_{j=1}^{r-1}\left(t_{k_{j}} \mathbf{f}_{j}\right)
\end{aligned}
$$


hold in $\mathbf{O}$. Since the letter $y$ is multiple in $\mathbf{u}$ and $\mathbf{v}$, we have that $r>2$ whenever (3.8) holds and $r>1$ otherwise. In view of Lemma 3.3,

$$
\mathbf{O}\left\{x^{2} y \prod_{j=1}^{r-1}\left(t_{k_{j}} \mathbf{f}_{j}\right) \approx x y x \prod_{j=1}^{r-1}\left(t_{k_{j}} \mathbf{f}_{j}\right)\right\}=\mathbf{O}\{\gamma\}
$$

where $\gamma=\gamma_{r-2}$ whenever (3.8) holds and $\gamma=\gamma_{r-1}^{\prime}$ whenever (3.12) holds. Therefore, $\mathbf{O}\{\mathbf{u} \approx \mathbf{v}\}$ satisfies $\gamma$. On the other hand, the identities

$$
\begin{aligned}
\mathbf{w} & =\mathbf{v}^{\prime} \mathbf{a} x y \mathbf{b} \mathbf{v}^{\prime \prime} & & \\
& \approx \mathbf{v}^{\prime} \mathbf{a} x^{2} y \mathbf{b} \mathbf{v}^{\prime \prime} & & \text { by }(1.1) \\
& \approx \mathbf{v}^{\prime} \mathbf{a} x y x \mathbf{b} \mathbf{v}^{\prime \prime \prime} & & \text { by the identity } \gamma \\
& \approx \mathbf{v}^{\prime} \mathbf{a} y x \mathbf{b} \mathbf{v}^{\prime \prime \prime} & & \text { by Lemma } 3.2 \\
& =\mathbf{v} & &
\end{aligned}
$$

hold in $\mathbf{O}\{\gamma\}$, whence $\mathbf{O}\{\mathbf{u} \approx \mathbf{v}\}=\mathbf{O}\{\mathbf{u} \approx \mathbf{w}, \gamma\}$.

So, we have proved that

$$
\text { either } \mathbf{O}\{\mathbf{u} \approx \mathbf{v}\}=\mathbf{O}\{\mathbf{u} \approx \mathbf{w}\} \text { or } \mathbf{O}\{\mathbf{u} \approx \mathbf{v}\}=\mathbf{O}\{\mathbf{u} \approx \mathbf{w}, \sigma\}
$$

for some $\sigma \in \Phi$. The identity $\mathbf{u} \approx \mathbf{w}$ is $(n-1)$-invertible. By the induction assumption, $\mathbf{O}\{\mathbf{u} \approx \mathbf{w}\}=\mathbf{O}\{\Sigma\}$ for some $\Sigma \subseteq \Phi$. It follows that $\mathbf{O}\{\mathbf{u} \approx \mathbf{v}\}=$ $\mathbf{O}\{\Sigma, \sigma\}$ whenever $\mathbf{O}\{\mathbf{u} \approx \mathbf{v}\}=\mathbf{O}\{\mathbf{u} \approx \mathbf{w}, \sigma\}$ and $\mathbf{O}\{\mathbf{u} \approx \mathbf{v}\}=\mathbf{O}\{\Sigma\}$ otherwise. Lemma 3.5 is proved.

Proof of Proposition 3.1. Let $\mathbf{V}$ be a subvariety of $\mathbf{O}$. If $\mathbf{E} \nsubseteq \mathbf{V}$ then $\mathbf{V}$ is finitely based by Lemmas 2.2 and 2.3(i). If $\mathbf{F} \nsubseteq \mathbf{V}$ then Lemmas 2.2 and 2.3(ii) imply that $\mathbf{V}$ is finitely based again. So, we may assume that $\mathbf{F} \vee \mathbf{E} \subseteq \mathbf{V}$. In view of Lemma 3.4, the variety $\mathbf{V}$ can be given within the variety $\mathbf{O}$ by a set of well-balanced identities $\Sigma$ together with the identity (1.4). According to Lemma 3.5, $\mathbf{O}\{\Sigma\}=\mathbf{O}\{\Psi\}$ for some $\Psi \subseteq \Phi$, where $\Phi$ has the same sense as in the proof of Lemma 3.5. Evidently, the identities $\alpha_{n+1}, \beta_{n+1}, \gamma_{n+1}$ and $\gamma_{n+1}^{\prime}$ follow from the identities $\alpha_{n}, \beta_{n}, \gamma_{n}$ and $\gamma_{n}^{\prime}$ respectively for any $n \in \mathbb{N}$. Therefore, the monoid variety given by the identity system $\Psi$ is finitely based. Then $\mathbf{V}$ is finitely based too.

\section{Proof of Theorem 1.1}

To prove Theorem 1.1, we need one auxiliary result.

Lemma 4.1. Let $\mathbf{V}$ be a monoid variety that contains the variety $\mathbf{F} \vee \mathbf{E}$ and satisfies the identity (1.1). If $\mathbf{V}$ does not contain $\mathbf{J}$ then $\mathbf{V}$ satisfies (1.5).

Proof. The words $\mathbf{p}$ and $\mathbf{q}$ are of the same type if $\mathbf{p}$ can be obtained from $\mathbf{q}$ by changing the individual exponents of letters and the second occurrence of $a$ is next to the first occurrence of $a$ in $\mathbf{p}$ if and only if the second occurrence of $a$ is next to the first occurrence of $a$ in $\mathbf{q}$ for any letter $a$. For example, the words $x y^{4} x z^{3} x^{5} y$ and $x y^{3} x^{4} z^{2} x^{2} y^{2}$ are of the same type. A word $\mathbf{w}$ is called reduced if $a \notin \operatorname{con}(\mathbf{p})$ whenever $\mathbf{w}=\mathbf{p} a^{2} \mathbf{q}$ for any $a \in \operatorname{con}(\mathbf{w})$. It is shown in [13, Section 3] that for any word $\mathbf{w}$ there is a unique reduced word $r(\mathbf{w})$ such that $\operatorname{sim}(\mathbf{w})=\operatorname{sim}(r(\mathbf{w}))$ and the words $\mathbf{w}$ and $r(\mathbf{w})$ are of the same type. This implies that the relation $\tau$ given by $\mathbf{u} \tau \mathbf{v}$ if and only if $r(\mathbf{u})=r(\mathbf{v})$ is a congruence on $F^{1}$. Let $W \leq$ denote the set of all subwords of a set of words $W$. 
In view of $[13$, Theorem $5.1(\mathrm{x})]$, the variety $\mathbf{J}$ is generated by the monoid $S_{\tau}\left(\left\{x z y x^{k} t y^{\ell} \mid k, \ell \in \mathbb{N}\right\} \leq\right)$. Then a word $\mathbf{u}$ in $\left\{x z y x^{k} t y^{\ell} \mid k, \ell \in \mathbb{N}\right\} \leq$ is not a $\tau$-term for $\mathbf{V}$ by Lemma 2.6. This means that $\mathbf{V}$ satisfies an identity $\mathbf{u} \approx \mathbf{v}$ with $(\mathbf{u}, \mathbf{v}) \notin \tau$. Since $\mathbf{F} \vee \mathbf{E} \subseteq \mathbf{V}$, Theorem 5.1(vii) and Fact 5.3(ii) of [13] imply that the word $\mathbf{u}$ contains a block with two distinct letters. Then $\mathbf{u} \in$ $\left\{x z y x^{k} t y^{\ell}, z y x^{m} t y^{\ell}, y x^{m} t y^{\ell} \mid k, \ell \in \mathbb{N}, m \geq 2\right\}$.

If $\mathbf{u}=y x^{m} t y^{\ell}$ then $\mathbf{v}=x^{p} y x^{q} t y^{r}$ for some $p, r \in \mathbb{N}$ and $q \geq 0$ by Lemma 2.4. It follows that $\mathbf{V}$ satisfies $x z y x^{m} t y^{\ell} \approx x z \mathbf{v}$, whence the word $x z y x^{m} t y^{\ell}$ is not a $\tau$-term for $\mathbf{V}$. If $\mathbf{u}=z y x^{m} t y^{\ell}$ then $\mathbf{v}=z x^{p} y x^{q} t y^{r}$ for some $p, r \in \mathbb{N}$ and $q \geq 0$ by Lemma 2.4. It follows that $\mathbf{V}$ satisfies $x z y x^{m} t y^{\ell} \approx x \mathbf{v}$, whence the word $x t y x^{m} z y^{\ell}$ is not a $\tau$-term for $\mathbf{V}$ again. So, we may assume that $\mathbf{u}=x z y x^{k} t y^{\ell}$. Then $\mathbf{v}=x t x^{p} y x^{q} t y^{r}$ for some $p, r \in \mathbb{N}$ and $q \geq 0$ by Lemma 2.4. If $q \geq 1$ then $\mathbf{V}$ satisfies the identities

$$
x z y x t y \stackrel{(1.1)}{\approx} x z y x^{k} t y^{\ell} \approx x t x^{p} y x^{q} t y^{r} \stackrel{(1.1)}{\approx} x t x y x t y,
$$

and we are done. If $q=0$ then the identities

$$
x z y x t y \stackrel{(1.1)}{\approx} x z y x^{k+1} t y^{\ell} \approx x t x^{p} y x t y^{r} \stackrel{(1.1)}{\approx} x t x y x t y,
$$

hold in $\mathbf{V}$, and we are done again.

Proof of Theorem 1.1. Let $\mathbf{V}$ be a limit variety within the class $\mathbf{A}_{\text {com. }}$. If $S(x y x) \in$ $\mathbf{V}$ then $\mathbf{V} \in\{\mathbf{L}, \mathbf{M}\}$ by [7, Theorem 3.2]. Suppose now that $S(x y x) \notin \mathbf{V}$. In view of Lemma 2.1, $\mathbf{V}$ satisfies one of the identities (1.1) or (2.1). By symmetry, we may assume without any loss that $\mathbf{V}$ satisfies (1.1). It is well known and can be easily verified that every variety from $\mathbf{A}_{\text {com }}$ satisfies the identity $x^{n} y^{n} \approx y^{n} x^{n}$ for some $n \in \mathbb{N}$. This identity together with (1.1) implies (1.2). According to Lemmas 2.2 and 2.3, $\mathbf{F} \vee \mathbf{E} \subseteq \mathbf{V}$. Suppose that $\mathbf{V} \neq \mathbf{J}$. Then Lemma 4.1 implies that $\mathbf{V}$ satisfies the identity (1.5). Thus, $\mathbf{V} \subseteq \mathbf{O}$. We obtain a contradiction with Proposition 3.1.

Theorem 1.1 is proved.

Acknowledgments. The author is sincerely grateful to Professor Boris Vernikov for his assistance in the writing of the manuscript, to Dr. Edmond W.H. Lee for several valuable remarks for improving the paper and to an anonymous referee for the suggestion of Lemma 4.1 and helpful comments.

\section{REFERENCES}

[1] S.V.Gusev, A new example of a limit variety of monoids, Semigroup Forum, accepted, DOI: 10.1007/s00233-019-10078-1.

[2] S.V.Gusev and B.M.Vernikov, Chain varieties of monoids, Dissertationes Math., 534 (2018), $1-73$.

[3] M.Jackson, Finiteness properties of varieties and the restriction to finite algebras, Semigroup Forum, 70 (2005), 154-187.

[4] M.Jackson and O.Sapir, Finitely based, finite sets of words, Int. J. Algebra and Comput., 10 (2000), 683-708.

[5] P.A.Kozhevnikov, On nonfinitely based varieties of groups of large prime exponent, Commun. Algebra, 40 (2012), 2628-2644.

[6] E.W.H.Lee, Finitely generated limit varieties of aperiodic monoids with central idempotents, J. Algebra and its Applications, 40 (2009), 779-796.

[7] E.W.H.Lee, Maximal Specht varieties of monoids, Moscow Math. J., 12 (2012), 787-802.

[8] E.W.H.Lee, On certain Cross varieties of aperiodic monoids with commuting idempotents, Results in Math., 66 (2014), 491-510. 
[9] E.W.H.Lee and J.R.Li, Minimal non-finitely based monoids, Dissertationes Math., 475 (2011), 1-65.

[10] P.Perkins, Bases for equational theories of semigroups, J. Algebra, 11 (1969), 298-314.

[11] G.Pollák, On two classes of hereditarily finitely based semigroup identities, Semigroup Forum, 25 (1982), 9-33.

[12] O.B.Sapir, Lee monoids are nonfinitely based while the sets of their isoterms are finitely based, Bull. Aust. Math. Soc., 97, No. 3 (2018), 422-434.

[13] O.B.Sapir, Limit varieties of J-trivial monoids, to appear; available at: https://arxiv.org/abs/2003.09950v2.

[14] L.N.Shevrin and M.V.Volkov, Identities of semigroups, Izv. VUZ. Matematika, No. 11 (1985), 3-47 [Russian; Engl. translation: Soviet Math Izv. VUZ, 29, No. 11 (1985), 1-64].

[15] M.V.Volkov, The finite basis problem for finite semigroups, Sci. Math. Jpn., 53 (2001), 171199.

[16] S.L.Wismath, The lattice of varieties and pseudovarieties of band monoids, Semigroup Forum, 33 (1986), 187-198.

[17] W.T.Zhang and Y.F.Luo, A new example of limit variety of aperiodic monoids, to appear; available at: https://arxiv.org/abs/1901.02207.

Ural Federal University, Institute of Natural Sciences and Mathematics, Lenina 51, 620000 Ekaterinburg, Russia

E-mail address: sergey.gusb@gmail.com 